\title{
Influence of Thermal Cycling in a Fe-Mn-Si-Cr Shape Memory Alloy
}

\author{
R.B. Pérez-Sáez, M.L. Nó* and J. San Juan \\ Dpto. Física Mat. Condensada, Fac. Ciencias, Univ. Pais Vasco, Apdo. 644, 48080 Bilbao, Spain \\ * Dpto. Física Aplicada II, Fac. Ciencias, Univ. Pais Vasco, Apdo. 644, 48080 Bilbao, Spain
}

\begin{abstract}
The evolution of the martensitic transformation with the thermal cycling (transformation temperatures and transformed fraction) has been studied in a $\mathrm{Fe}-29 \mathrm{Mn}-6 \mathrm{Si}-6 \mathrm{Cr}$ (wt.\%) shape memory alloy. The study was done by internal friction and modulus measurements, using an inverted torsion pendulum at $1 \mathrm{~Hz}$. The internal friction peaks associated with the martensitic transformation are clearly observed in the spectra. This way the transformation temperatures and their evolution during cycling was determined. The study of the transformed fraction was carried out using the peak area. The experimental results and the behaviour of these alloys with the thermal cycling are discussed in the frame of a model of dislocation creation during cycling.
\end{abstract}

\section{INTRODUCTION}

The origin of the shape memory effect in $\mathrm{Fe}-\mathrm{Mn}-\mathrm{Si}(-\mathrm{Cr})(-\mathrm{Ni})$ alloys is the martensitic transformation (MT) from $\gamma$-austenite (f.c.c.) to $\varepsilon$-martensite (h.c.p.).

Typical $\mathrm{Cu}$-based and NiTi alloys exhibit a thermoelastic MT. In contrast in these iron based alloys the transformation is non thermoelastic. This means that during the MT a certain amount of local plastic deformation is induced in the matrix and the boundaries between the martensite and the austenite tend to become locked [1]. Then, the reverse transformation from the martensite to the austenite is more difficult and it is not possible to accommodate a macroscopic deformation using martensite previously formed. These facts impose the characteristics and the properties of these alloys: very large thermal hysteresis $\left(120^{\circ} \mathrm{C}\right.$ aprox.), and a shape memory effect due to the reverse transformation of stress-induced $\varepsilon$-martensite.

As a consequence of the large thermal hysteresis, these alloys do not exhibit superelastic effect because $\mathbf{M}_{\mathrm{d}}<\mathrm{A}_{\mathbf{f}}$. However, the large thermal hysteresis is a good property in some special applications for which a stability in large ranges of temperatures is required. Besides, their low cost and good workability must be considered.

In this work the evolution of the MT in a Fe-29Mn-6Si-6Cr alloy as a function of the thermal cycling has been studied. This alloy has the Néel temperature $\left(T_{N}\right)$ close to Ms because of the quite high concentration of manganese. The aim of this study is to show the effect of this fact, and to compare the results with a previous work [2] in a Fe-16Mn-9Cr-5Si-4Ni alloy where there is no interaction between the MT and the magnetic transition.

\section{EXPERIMENTAL METHODS}

The alloy of Fe-28.7Mn-6.1Si-6.0Cr (wt.\%) was produced by the NKK Corporation and was hot rolled into a plate $0.5 \mathrm{~mm}$ thick. Samples of $50 \times 5 \times 0.5 \mathrm{~mm}^{3}$ were cut for internal friction and modulus measurements. The sample was annealed for $1 \mathrm{~h}$ at $900^{\circ} \mathrm{C}$ in a helium atmosphere and ice-water quenched. According to previous works on similar samples [3,4], this treatment produces a recrystallization of the sample and consequently the austenitic phase should contain a very low density of dislocations. 
The internal friction and shear modulus measurements were carried out in an inverted torsion pendulum working at about $1 \mathrm{~Hz}$. and strain amplitude of $5 \times 10^{-6}$. Complete transformation cycles between $115 \mathrm{~K}$ and $550 \mathrm{~K}$ were performed with a heating-cooling rate of $100 \mathrm{~K} / \mathrm{h}$, starting and finishing always at high temperature in $\gamma$ phase.

\section{EXPERIMENTAL RESULTS}

In Fig. 1 the logarithmic decrement $(\delta)$ and the square of the resonance frequency $\left(\mathrm{f}^{2} \propto\right.$ modulus$)$ are plotted versus the temperature for a complete transformation cycle. The internal friction (IF) spectra show clearly the peaks associated with the forward MT $(\gamma-\varepsilon)$ during cooling and with the reverse MT ( $\varepsilon-\gamma)$ during heating. We can point out the large thermal hysteresis characteristic of the non thermoelastic MT. In contrast to the behaviour of the $\mathrm{Cu}-\mathrm{Zn}-\mathrm{Al}$ and $\mathrm{Ti}-\mathrm{Ni}$ alloys [5], the $\mathrm{IF}$ background at low temperature is as low as in the $\gamma$ phase at high temperature. This fact could be due to the small amount of transformed martensite or to the low mobility of the martensitic interfaces [6].

The modulus curves show an increase when the forward MT take place. This behaviour is also very different from that observed in the thermoelastic shape memory alloys where the shear modulus shows a minimum associated to the MT. The explanation of this effect is related to two different aspects. On one hand, in this iron based alloys there is no experimental evidence of elastic constants softening around the transformation such as is observed in the thermoelastic shape memory alloys. On the other hand, even if some softening could take place, it will be masked by the strong internal stresses developped during the formation and growing of the martensitic phase, that produce a progressive hardening of the microstructure and consequently a strong increase in the dynamic modulus.

Finally, a sharp decrease in the modulus, associated with the paramagnetic-antiferromagnetic transition of the $\gamma$ phase, is observed at around $230 \mathrm{~K}$.

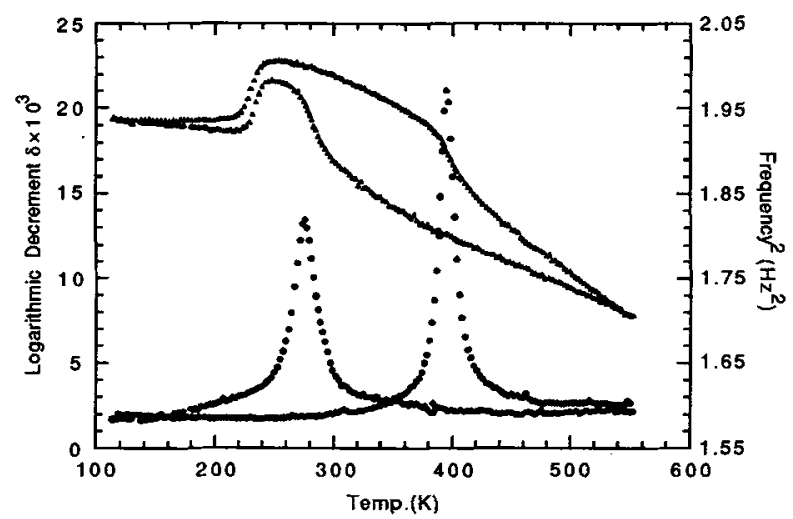

Fig.1 Logarithmic decrement $\delta(\bullet)$ and square of the frequency $(\Delta)$, versus temperature during the fith cycle of the transformation (temperature rate $100 \mathrm{~K} / \mathrm{h}$ ).

\subsection{Evolution of Internal friction spectra with the thermal cycling}

In Fig. 2 we have plotted the IF spectra of the $2^{\text {nd }}, 24^{\circ}$ and $57^{\circ}$ cycles. During cycling, a strong decrease of the peak height associated with the reverse MT is observed. The peak associated with the forward MT decreases less strongly but it also undergoes a sharpening. Besides, the peak temperature shifts towards lower temperatures (Fig.3) rather quickly during the first cycles and shows a tendency to a further stabilization. It is interesting to remark that the the reverse MT peak shifts as a whole while for the forward transformation peak the shifting takes place mainly in the high temperature side (Fig.4). The low temperature side of this peak does not shift and then a sharpening and a decrease of the peak is produced as we have 
already commented. Nevertheless, the peak height is not a good parameter to follow the evolution of the transformation during cycling, because it is related to the transformation rate. Probably the best parameter should be the peak area that is related to the amount of the transformed phase during the cycle [2]. In Fig.5, the average peak area for the cooling and heating runs has been plotted (in arbitrary units) as a function of the number of cycles. A clear decrease of the peak area is observed during cycling.
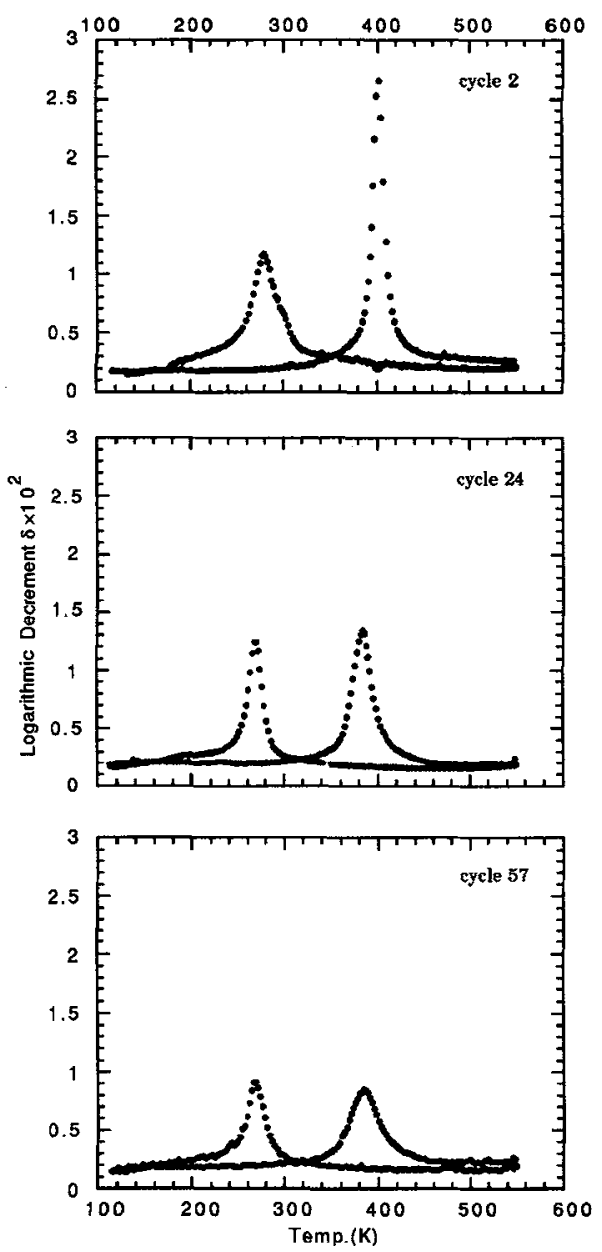

Fig.2 Internal friction spectra for three different cycles.

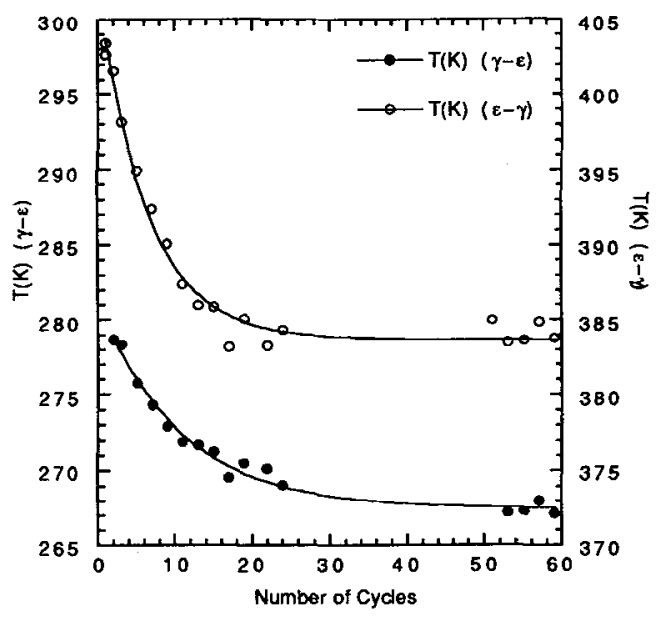

Fig.3 Evolution of the transformation temperatures during the thermal cycling:

- Shift of the forward transformation peak $(\gamma-\varepsilon)$

OShift of the reverse transformation peak $(\varepsilon-\gamma)$

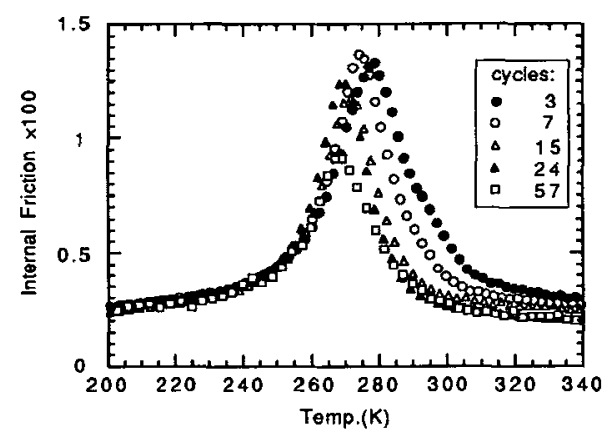

Fig.4 Internal friction peak of the forward transformation for several number of cycles.

\subsection{Evolution of the modulus with the thermal cycling}

In Fig. 6 we have plotted the modulus curves for several transformation cycles whose number of cycle is indicated. The curves have been vertically shifted in order to the picture clarity. Actually, the modulus value at high temperatures (in $\gamma$ phase) is more or less the same. 
We have to point out that a strong decrease of the area inside the modulus cycle is produced with the thermal cycling. Besides, the modulus variation linked to the forward and reverse transformation is shifted toward low temperature in a similar way to the shifting of the IF peaks.

The modulus change associated to the magnetic transition allows us to estimate the Néel temperature. Its evolution with the thermal cycling is shown in Fig.7 where a slow increase in Néel temperature is observed.

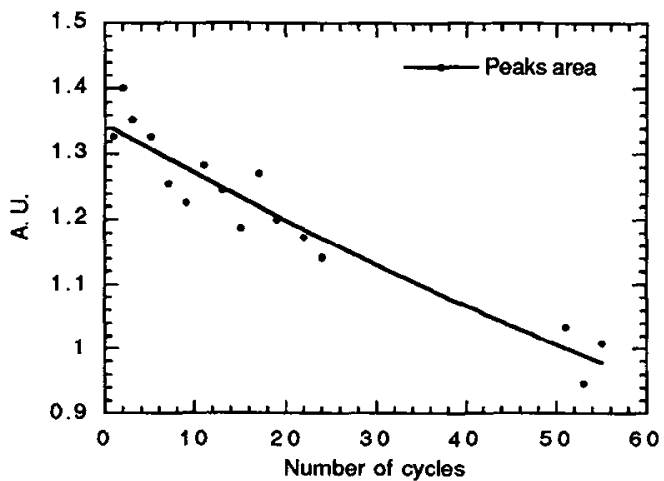

Fig.5 Evolution of the peaks area during the thermal cycling.

Fig.6 Modulus curves for several number of thermal cycles. (Vertically shifted to improve picture clarity).
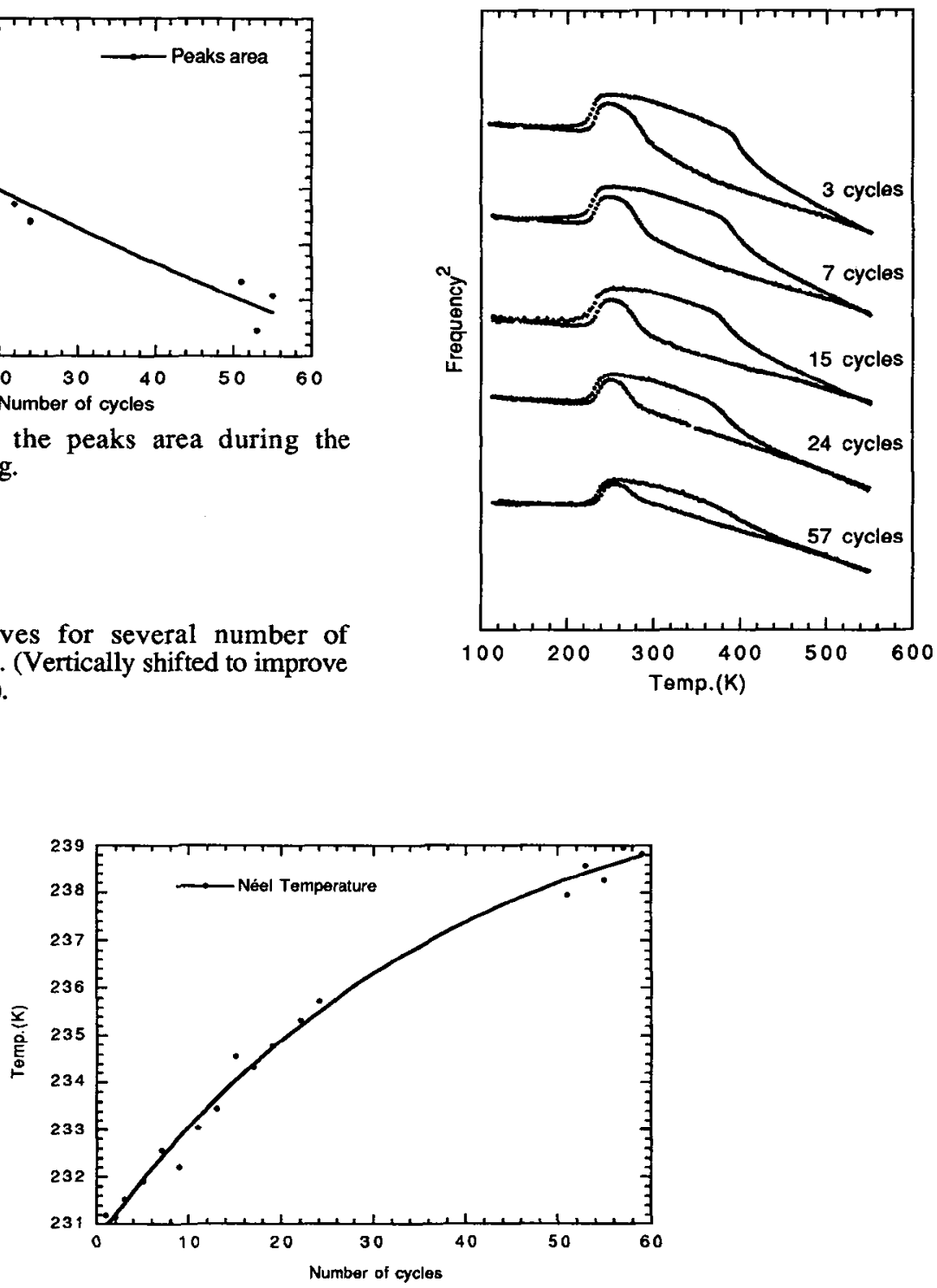

Fig.7 Evolution of estimated Néel temperature with thermal cycling. 


\section{DISCUSSION}

It is well known that the $\varepsilon$-martensite appears from the stacking fault between the Shockley partials in which the $\langle 101\rangle\{111\}$ perfect dislocations are dissociated. Besides, in these alloys the MT is not thermoelastic and some transformation strain is accommodated plastically producing new dislocations on the [111\} planes in which the transformation are accumulated [7]. This plastic accommodation of the deformation hinders the growing of the martensite variants in such a way that the transformation is stopped when the transformed martensite is only $15 \%$ [3].

Another effect to take into account in these alloys is the stabilization of the $\gamma$ phase when the Néel temperature $\left(T_{N}\right)$ is reached. At this point, the $\gamma$ phase undergoes a transition from a paramagnetic state to an antiferromagnetic one. The decrease of the free energy characteristic of this transition is responsible for the stabilization of the $\gamma$ phase that could produce different effects on the martensitic transformation [8]:

1-If $T_{N}>M_{S}$, the magnetic transition could hinder completely the martensitic transformation.

2-If $\mathrm{M}_{\mathrm{f}}<\mathrm{T}_{\mathrm{N}}<\mathrm{M}_{\mathrm{s}}$, the martensitic transformation could be stopped before it is normally finished.

3-If $T_{N}<M_{S}$ there will be no interaction between the magnetic transition and the MT.

The whole set of results can be interpreted as follows. During the thermal cycling the dislocation density in $\gamma$ phase increases and the stress field enhances the elastic term of the free energy of the martensite formation that becomes more and more difficult. Consequently, a higher driving force is needed, leading to a temperature decrease of the direct transformation (Fig.3). For the same reason, the temperatures of the reverse transformation also decrease (Fig.3).

In addition, the increase of the dislocation density during the cycling means that in the next cycle there will exist a higher number of martensite nucleation sites (<101 > dislocations) and an increase of volume fraction of the transformed martensite should be expected, such as we observed in a similar alloy [2]. Nevertheless, the experimental results do not confirm this behaviour because in Fig.5 the IF peak area (proportional to the transformed volume fraction) decrease with thermal cycling. The reason why we observe this behaviour is because in this alloy the Néel temperature is very close to the martensitic transformation $\left(M_{\mathrm{f}}<\mathrm{T}_{\mathrm{N}}\right)$ and then the magnetic transition stops the MT, such as we have already commented. Besides, $M_{S}$ decreases (Fig.3) and $T_{N}$ increases (Fig.7) with the cycling, in such a way that the magnetic transition stops the MT the more and more early. This blockade of the MT is seen in Fig.4 when the IF peak shifts with the thermal cycling and decreases because it becomes piled up against the low temperature side that is the lower limit imposed by the magnetic transition.

In the previous work in a Fe-16Mn-9Cr-6Si-5 Ni (wt.\%) alloy [2] we observed indeed an increase of the transformed volume fraction of the martensite with cycling, but in this alloy the Néel Temperature $T_{N}$ was far enough from the martensitic transformation to produce any effect.

\section{CONCLUSIONS}

We have shown the necessity of a previous cycling in order to stabilize the transformation temperatures in this kind of alloys.

We have verified the importance of the relative position of $T_{N}$ and the martensitic transformation in order to control the amount of transformed phase and its evolution with the thermal cycling. This aspect is just responsible of the different behaviour observed between the alloys studied in this work and the one studied in a previous work [2].

\section{ACKNOWLEDGEMENT}

This work has been carried out with the financial support of the Spanish "Comision Interministerial de Ciencia y Tecnología (CICyT)" (project nº MAT92-0353). R.B. Pérez-Sáez wishes to acknowledge the Basque Government for providing him a pre-doctoral scholarship.

\section{REFERENCES}

[1] G.B. Olson and M. Cohen, Scripta Metall. 9 (1975) 1247-1254.

[2] R.B. Pérez-Sáez, M.L. Nó and J. San Juan, J. of Alloys and Compounds 211/212 (1994) 212-215.

[3] L. Federzoni, D. Gex, Q. Gu, G. Guénin, D. Labrosse, M. Mantel and J. Van Humbeeck, Proc. of the Inter. Conf. on Martensitic Transformations, Monterey, Ca, USA 1992; (Inst. for Advanced Studies, Monterey, 1993), pp. 1175-1180. 
[4] Q. Gu, Ph. D. Thesis: "Martensitic Transformation and Shape Memory Effect in Fe-Mn-Si Shape Memory Alloys", Katholieke Universiteit Leuven (1994).

[5] S. Koshimizu, Ph. D. Thesis No 416: "Etude par Mesure du Frottement Interieur d'une Transformation Martensitique", Lausanne EPFL (1981).

[6] J.E. Bidaux, Ph. D. Thesis No 761: "Etude par Methodes Acoustiques de la Transition de Phase HCCFC du Cobalt", Lausanne EPFL (1988).

[7] M. Sade and E. Hornbogen, The Martensitic Transformation in Science and Technology (ECOMAT89), (Eds. E. Hornbogen and N. Jost, Verlag, 1989) pp. 249-256.

[8] A. Sato, Inter. Meeting on Advanced Materials, Tokyo, May 31-June 3, 1988 (MRS, Pittsburgh 1989) vol. 9, pp. 431-445. 